\title{
Lung Involvement in Rheumatic Disease: Introduction
}

\author{
Demosthenes Bouros $^{a} \quad$ Spyros Papiris $^{b}$ Vincent Cottin ${ }^{c}$ \\ ${ }^{a}$ Academic Department of Pneumonology, Sotira General Hospital for Chest Diseases, and ${ }^{\mathrm{b}}$ 2nd Pulmonary \\ Medicine Department, 'Attikon' University Hospital, Athens, Greece; 'Service de Pneumologie, Louis Pradel Hospital, \\ Reference Center for Rare Pulmonary Diseases, Claude Bernard University Lyon 1, Lyon, France
}

Lung involvement in autoimmune rheumatic diseases is a common manifestation and a major cause of mortality and morbidity. Involvement may include lung parenchyma, airways, pleura, vessels and respiratory muscles. Main differential diagnoses include rheumatic disease manifestation, drug-induced manifestation or infection.

Interstitial lung disease (ILD) in connective tissue disease (CTD) can be characterized using the classification of the idiopathic interstitial pneumonias. CTDs as a whole may develop all histologic patterns observed in idiopathic interstitial pneumonias. Systemic sclerosis is most frequently associated with ILD and, in most of these patients, ILD manifests as a histological pattern of nonspecific interstitial pneumonia. Conversely, in rheumatoid arthritis, the pattern of ILD seems to be most often usual interstitial pneumonia. Histologic patterns may be combined, as exemplified by the frequent association of ILD with lymphocytic bronchiolitis in rheumatoid arthritis. Occasionally, lung involvement can present before the diagnosis of CTD, especially in idiopathic inflammatory myopathy and rheumatoid arthritis.

In order to decrease mortality, there is a need for early, exact diagnosis and effective treatment. Cooperation of experienced physicians for possible complications, including infections, drug-induced adverse effects, direct or indirect pulmonary or cardiovascular complications, is of paramount importance for the best management.
Heterogeneity in the extent and progression of lung fibrosis probably reflects differences in the underlying pathogenic mechanisms. A growing understanding of the key pathogenic drivers of lung fibrosis and of CTDs (including the role of tobacco smoking) might lead to the development of more effective targeted therapies.

The key goals of the clinical assessment of patients with both ILD and CTD are the detection of ILD and prognostic evaluation to determine which patients should be treated and how.

Recent developments have moved the field forward. Several studies have provided significant advances, especially regarding HRCT-pathologic correlations, prediction of the outcome based on imaging and/or pathology, and the relevance of subtle clinical, serologic and morphologic imaging/pathological findings not fulfilling the criteria for CTDs (a condition now called interstitial pneumonia with autoimmune features'). Demonstration of the efficacy of antifibrotic drugs to slow disease progression in idiopathic pulmonary fibrosis has raised hope that these or other drugs might be effective in fibrotic ILD associated with CTD and foster clinical research.

This thematic series sets the scene of CTD principles for a series of papers that will focus on the investigation of lung involvement in rheumatologic disease, the investigation of CTD in ILD, biomarkers, treatment considerations, acute exacerbation and monitoring of lung involvement in rheumatologic disease from authorities in the field.

\section{KARGER 125}

(c) 2015 S. Karger AG, Base

0025-7931/15/0901-0001\$39.50/0
Prof. Demosthenes Bouros, MD, PhD, FERS, FCCP

Academic Department of Pneumonology

Sotira Hospital for Chest Diseases

Messogion Avenue 152, GR-11527 Athens (Greece)

E-Mail dbouros@med.uoa.gr 